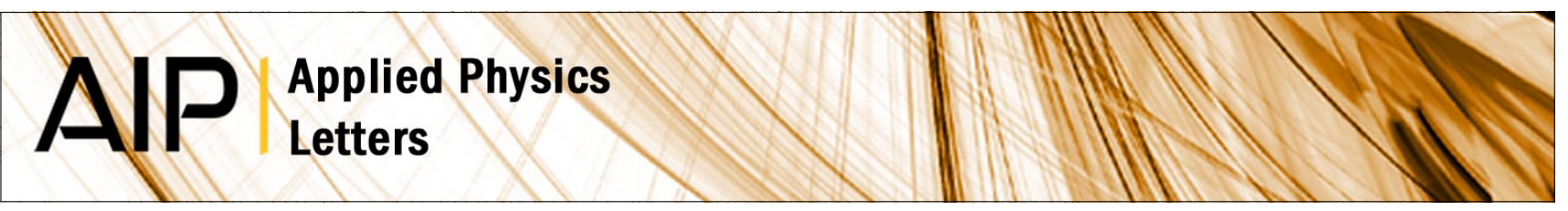

\title{
Far infrared photoconductivity in a silicon based material: Vanadium supersaturated silicon
}

E. García-Hemme, R. García-Hernansanz, J. Olea, D. Pastor, A. del Prado et al.

Citation: Appl. Phys. Lett. 103, 032101 (2013); doi: 10.1063/1.4813823

View online: http://dx.doi.org/10.1063/1.4813823

View Table of Contents: http://apl.aip.org/resource/1/APPLAB/v103/i3

Published by the AIP Publishing LLC.

Additional information on Appl. Phys. Lett.

Journal Homepage: http://apl.aip.org/

Journal Information: http://apl.aip.org/about/about_the_journal

Top downloads: http://apl.aip.org/features/most_downloaded

Information for Authors: http://apl.aip.org/authors

\section{ADVERTISEMENT}
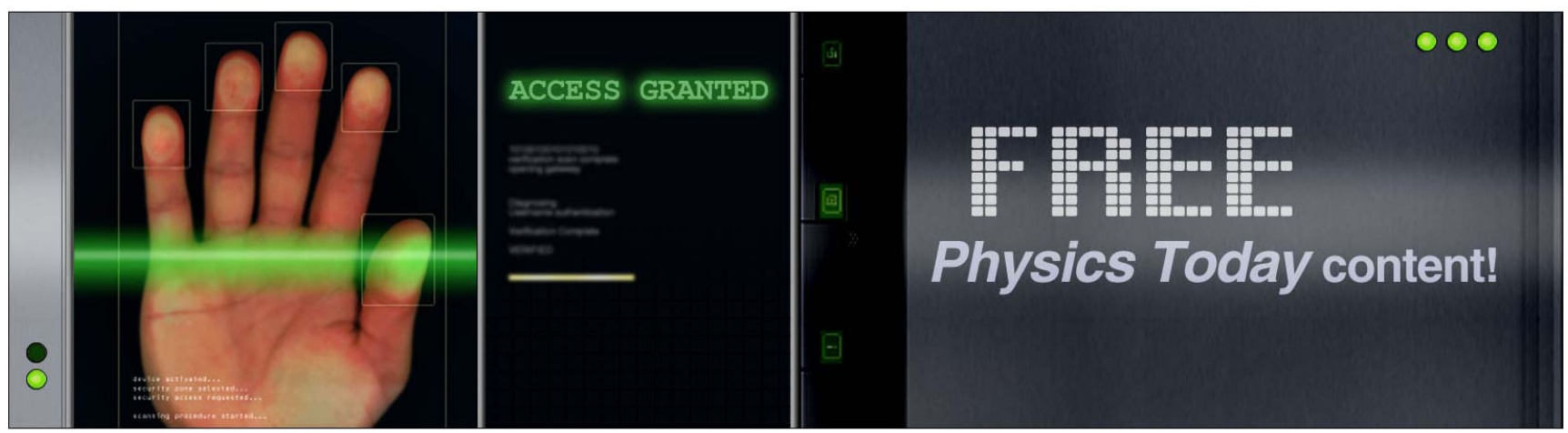


\title{
Far infrared photoconductivity in a silicon based material: Vanadium supersaturated silicon
}

\author{
E. García-Hemme, ${ }^{1,2}$ R. García-Hernansanz, ${ }^{1,2}$ J. Olea, ${ }^{2,3}$ D. Pastor, ${ }^{1,2,3}$ A. del Prado, ${ }^{1,2}$ \\ I. Mártil, ${ }^{1,2}$ and G. González-Díaz ${ }^{1,2}$ \\ ${ }^{1}$ Dept. de Física Aplicada III (Electricidad y Electrónica), Univ. Complutense de Madrid, \\ 28040 Madrid, Spain \\ ${ }^{2}$ CEI Campus Moncloa, UCM-UPM, 28040 Madrid, Spain \\ ${ }^{3}$ Instituto de Energía Solar, E.T.S.I. de Telecomunicación, Univ. Politécnica de Madrid, 28040 Madrid, Spain
}

(Received 12 June 2013; accepted 27 June 2013; published online 15 July 2013)

\begin{abstract}
We have analyzed the spectral sub-bandgap photoresponse of silicon ( $\mathrm{Si}$ ) samples implanted with vanadium (V) at different doses and subsequently processed by pulsed-laser melting. Samples with V concentration clearly above the insulator-metal transition limit show an important increase of the photoresponse with respect to a Si reference sample. Their photoresponse extends into the far infrared region and presents a sharp photoconductivity edge that moves towards lower photon energies as the temperature decreases. The increase of the value of the photoresponse is contrary to the classic understanding of recombination centers action and supports the predictions of the insulator-metal transition theory. @ 2013 AIP Publishing LLC. [http://dx.doi.org/10.1063/1.4813823]
\end{abstract}

Silicon ( $\mathrm{Si}$ ) has been the key material since the beginning of the microelectronic era. However, there are specific applications in which $\mathrm{Si}$ cannot compete with other materials. For example, in the field of the infrared detection $\mathrm{PbSe}^{1}$ or $\mathrm{InSb}^{2}$ are more suitable than $\mathrm{Si}$. For this reason, the fabrication of an infrared photodetector based on $\mathrm{Si}$ would be of a great interest and the advantages are clear: any Si based material would be easily integrated in the very mature Si microelectronics industry.

To attain these goals, several approaches are now in progress at different laboratories worldwide. Si supersaturated with chalcogens prepared by laser irradiation in $\mathrm{SF}_{6}$ atmosphere has exhibited strong sub gap absorption. ${ }^{3}$ Photodetectors build-up with Si highly doped with $\mathrm{S}$ and Se by means of ion implantation and subsequently pulsed laser melted (PLM) have shown extended infrared photoresponse below the Si bandgap with high gain levels. ${ }^{4}$ In previous papers, we have shown that $\mathrm{Si}$ supersaturated with $\mathrm{Ti}$ by ion implantation and then PLM treated shows unusual optoelectronic characteristics. ${ }^{5-7}$ Electrical transport properties of these materials have been well explained for both Ti concentrations below and above the insulator-metal transition, also known as Mott transition. ${ }^{8}$

Normally the introduction of deep level impurities drastically reduces the carrier lifetime since these impurities act as non-radiative recombination (NRR) centers, enhancing the multiphonon emission. ${ }^{9}$ However, at very high impurity concentrations, the electron wave functions would overlap producing a delocalization of the states associated to the impurities, just as it happens with the electron wave functions in the valence band (VB) and in the conduction band (CB). The concentration that determines the insulator-metal transition (Mott limit) has been theoretically calculated to be approximately $6 \times 10^{19} \mathrm{~cm}^{-3} .^{10}$ This value is in agreement with experimental results. ${ }^{11,12}$ Once this limit is achieved, the reduction of the NRR is possible and an increase of the charge carrier lifetime may take place, as explained in the configuration diagram theory. ${ }^{10}$
As we have proved recently by means of lifetime measurements, Ti supersaturated $\mathrm{Si}$ samples have exhibited lifetime recovery, in agreement with the previous arguments. ${ }^{13}$ These results demonstrated that once the Mott transition is achieved, the delocalization of the states associated to the impurities occurs and the so-called intermediate band (IB) is formed at this supersaturated region. More recently, we have proved again the lifetime recovery of these samples by means of spectral photo response measurements. ${ }^{14}$

In this letter, we present the photoelectronic and structural characteristics of Si supersaturated with vanadium $(\mathrm{V})$. $\mathrm{V}$ is a well-known deep center in $\mathrm{Si}^{15}$ and as a consequence, a possible candidate to form an IB under adequate experimental conditions.

Samples $1 \times 1 \mathrm{~cm}^{2}$ in size of n-type $\mathrm{Si}$ (111) with a thickness of $300 \mu \mathrm{m}\left(\rho \approx 200 \Omega \mathrm{cm}, \mu \approx 1500 \mathrm{~cm}^{2} \mathrm{~V}^{-1} \mathrm{~s}^{-1}\right.$, $\mathrm{n} \approx 2.2 \times 10^{13} \mathrm{~cm}^{-3}$ at room temperature) were implanted at $32 \mathrm{keV}$ with $\mathrm{V}^{51}$ at two different doses $\left(10^{13}\right.$ and $\left.10^{16} \mathrm{~cm}^{-2}\right)$ using a $7^{\circ}$ tilt angle. Subsequently, the implanted samples were PLM processed at $1 \mathrm{~J} \mathrm{~cm}^{-2}$ with a $\mathrm{KrF}$ excimer laser $(248 \mathrm{~nm})$ at IPG Photonics. Also, some Si samples were implanted with $\mathrm{Si}$ at $170 \mathrm{keV}$ and $10^{16} \mathrm{~cm}^{-2}$ dose and subsequently processed by PLM at $1 \mathrm{~J} \mathrm{~cm}^{-2}$. We have chosen $170 \mathrm{keV}$ for the Si implanted Si samples because our objective is to obtain a more defective and thicker layer after the implantation and PLM process for comparative purposes.

Four Ti/Al triangular contacts were deposited on the corners of the samples by means of the e-beam evaporation. The spectral photoconductance of the samples for energies below the bandgap was analyzed using the van der Pauw setup. With this setup, a fixed current was injected through two adjacent contacts and the $\mathrm{AC}$ voltage generated over the opposite two contacts was measured, while a monochromatic chopped light impinged the samples. A TMc300 Bentham monochromator with a Nernst filament source was used as infrared monochromatic source. The intensity of the light was calibrated with a Bentham pyrometric detector. Measurements were carried out at $90 \mathrm{~K}$ placing the samples 
inside a closed-cycle Janis cryostat and a vacuum pump was used to avoid moisture condensation at low temperature. The van der Pauw setup was fed with $1 \mathrm{~mA}$ DC current to avoid self-heating effects. Photovoltage measurements were carried out with a SR830 digital signal processing lock-in amplifier manufactured by Stanford Research Systems. Sheet conductance in darkness was measured using the same setup than in photovoltage measurements, but in this case the temperature ranged from $10 \mathrm{~K}$ to $300 \mathrm{~K}$. We have used a Keithley SCS 4200 model with four I-V source and measure units to perform the darkness sheet conductance measurements as a function of temperature.

To analyze the $\mathrm{V}$ depth profile, time-of-flight secondary ion mass spectrometry (ToF-SIMS) measurements were carried out in a ToF-SIMS IV model manufactured by IONTOF, with a $25 \mathrm{keV}$ pulsed $\mathrm{Bi}^{3+}$ beam at $45^{\circ}$ incidence. A $10 \mathrm{keV}$ voltage was used to extract the secondary ions generated and their time of flight from the sample to the detector was measured using a reflection mass spectrometer. The structural characterization of the samples was carried out by transmission electron microscopy (TEM) and electron diffraction (ED) patterns obtained with a Titan3 G2 working at $300 \mathrm{keV}$.

As the fabrication of $\mathrm{V}$ supersaturated $\mathrm{Si}$ is intended for infrared photodetector or solar cell applications, one of its key properties is the spectral photoresponse. Fig. 1 shows the results of the sheet conductance increase under illumination $\left(\frac{\Delta G_{\square}}{I_{0}}\right)$ normalized to the incident light power density $I_{0}$, as a function of the incident photon energy. The sheet conductance increase is directly related to the increase of the charge carrier concentrations with respect to the darkness equilibrium state, as it was previously shown in Ref. 14: $\Delta G_{\square}=q \mu \Delta n t$, where $q$ is the electron charge, $\mu$ is the charge carrier mobility, $\Delta n$ is the charge carrier concentration increase due to the illumination, and $t$ is the sample thickness. The $\frac{\Delta G_{\square}}{I_{0}}$ is presented for the $\mathrm{V}$ implanted samples

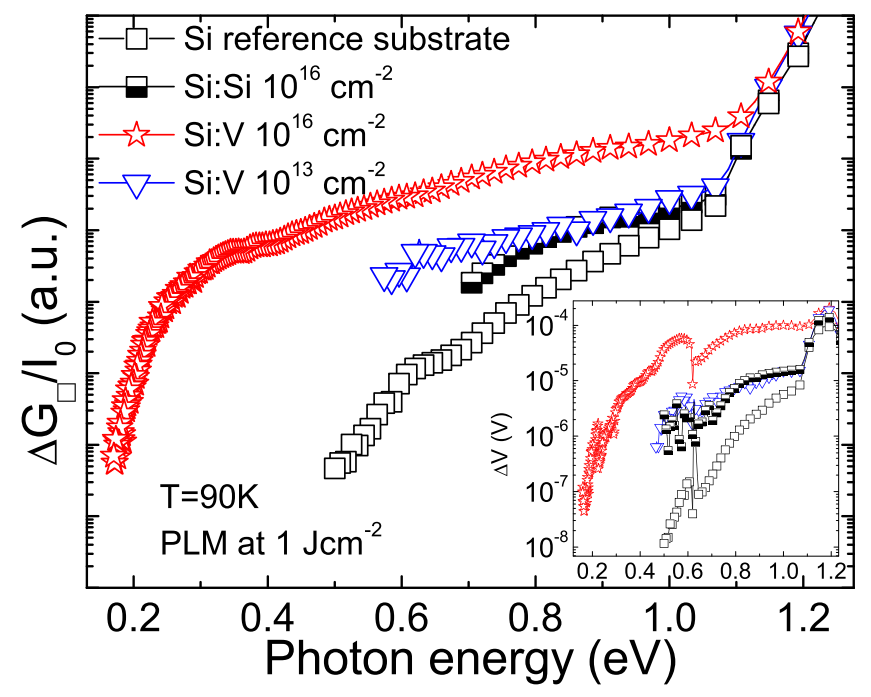

FIG. 1. Increase of the sheet conductance spectral response normalized to the impinging light power density as a function of the incident photon energy for the Si unimplanted reference sample, for the V implanted Si samples at $10^{13}$ and $10^{16} \mathrm{~cm}^{-2}$ doses and the Si implanted Si sample at $10^{16} \mathrm{~cm}^{-2}$ dose. Implanted samples were subsequently PLM processed at $1 \mathrm{~J} \mathrm{~cm}^{-2}$. Inset shows the AC voltage measured as a consequence of the chopped light that impinges the samples. Measurements were carried out at $90 \mathrm{~K}$. at $10^{13}$ and $10^{16} \mathrm{~cm}^{-2}$ doses and PLM at $1 \mathrm{~J} \mathrm{~cm}^{-2}$ and the $\mathrm{Si}$ implanted sample at $10^{16} \mathrm{~cm}^{-2}$ dose and PLM at $1 \mathrm{~J} \mathrm{~cm}^{-2}$. We have chosen two different $\mathrm{V}$ concentrations well below and above the theoretical Mott limit to clearly observe the possible effect related to the insulator-metal transition. Also, a reference silicon unimplanted substrate is presented for comparative purposes. In order to observe the different levels of the electrical noise for the different samples, the inset in Fig. 1 shows the AC voltage (darkness voltage-illumination voltage) measured directly by the lock-in amplifier. Due to the high noise, $\frac{\Delta G_{\square}}{I_{0}}$ is not represented below $0.6 \mathrm{eV}$ for the $\mathrm{V}$ implanted sample at $10^{13} \mathrm{~cm}^{-2}$ and $0.7 \mathrm{eV}$ for the $\mathrm{Si}$ implanted Si sample.

For all the samples, we can observe the abrupt increase of the $\frac{\Delta G_{\square}}{I_{0}}$ magnitude for photon energies over $1.1 \mathrm{eV}$, corresponding to the intrinsic charge carrier generation due to the $\mathrm{Si}$ band to band transitions. Concerning the Si reference substrate, we observe a minor photoresponse for infrared photon energies. This infrared photoresponse has been well explained in terms of carrier generation due to surface states. $^{16,17}$

For the $\mathrm{Si}$ implanted $\mathrm{Si}$ sample and the $\mathrm{V}$ implanted sample with the lowest dose $\left(10^{13} \mathrm{~cm}^{-2}\right)$, we observe a slightly higher photoresponse than for the Si reference substrate and an important increase of the electrical noise that has made it impossible to measure at lower photon energies. A very different behavior is observed in the case of the $\mathrm{V}$ implanted sample at $10^{16} \mathrm{~cm}^{-2}$. The photoresponse of this sample is at least one order of magnitude higher than the $\mathrm{Si}$ reference sample at $1 \mathrm{eV}$ photon energy and almost 4 orders of magnitude higher at $0.5 \mathrm{eV}$ photon energy. Its response is extended deep into the infrared region, presenting an abrupt front of $\frac{\Delta G_{\square}}{I_{0}}$ for energies starting below $0.2 \mathrm{eV}$. Moreover, the noise level is remarkably lower than in the sample implanted at $10^{13} \mathrm{~cm}^{-2}$ dose, and comparable to the noise level of the Si reference substrate. All these characteristics (electrical noise and different levels of photoresponse) will be discussed in the following paragraphs.

By ToF-SIMS, we have studied the $\mathrm{V}$ concentration depth profile of the implanted and PLM samples. For the sample implanted with the lowest dose $\left(10^{13} \mathrm{~cm}^{-2}\right)$, the Mott limit concentration $\left(6 \times 10^{19} \mathrm{~cm}^{-3}\right)$ is not reached. However, the sample implanted at $10^{16} \mathrm{~cm}^{-2}$ dose presents a $\mathrm{V}$ concentration over the theoretical Mott limit in a layer of about $100 \mathrm{~nm}$. Fig. S1 in supplemental material ${ }^{18}$ shows V depth profile for both samples. Therefore, according to the Mott transition theory, two different scenarios have been obtained as a function of the implanted dose, which explains the photoconductivity measurements shown in Fig. 1. In the case of the sample implanted at $10^{13} \mathrm{~cm}^{-2}$ dose, we have obtained a layer that presents a high $\mathrm{V}$ concentration which is below the theoretical Mott limit, and possibly the insulator-metal transition has not been achieved. Therefore, the observed infrared photoresponse of this sample is mainly due to $\mathrm{V}$ deep levels introduced in the $\mathrm{Si}$ lattice as it has been previously observed for iron deep levels in $\mathrm{Si}^{19}$ or $\mathrm{Zn}$ deep levels in $\mathrm{Si}^{20}$ Additionally, these $\mathrm{V}$ deep level impurities would act as an important source of localized recombination centers, increasing the electrical noise due to the recombination 
processes, ${ }^{21-23}$ which could explain the measurements for this sample in Fig. 1. In contrast, the sample implanted with the $10^{16} \mathrm{~cm}^{-2}$ dose shows a V concentration over the theoretical Mott limit in a $100 \mathrm{~nm}$ thick layer (Fig. S1). ${ }^{18}$ For this sample, the theoretical delocalization transition point has been surpassed and we could expect a reduction of the NRR processes at the $\mathrm{V}$ implanted layer. This reduction of the recombination processes could be related with the remarkable decrease of the electrical noise observed in the photoconductivity measurements of this sample (Fig. 1). Therefore, this result is consistent with the delocalization transition of the deep level impurities in the implanted layer. Moreover, surpassing the delocalization transition limit would imply the formation of a band of allowed states within the Si bandgap. The formation of this band would result in an increase of both the charge carrier lifetime and the absorption coefficient, due to sub-bandgap optical transitions involving this extra band. Both mechanisms would lead to an increase of the photoresponse, being the influence of the absorption coefficient dominant near the optical transition edge. The intense increase of the photoresponse observed for the sample implanted at a $10^{16} \mathrm{~cm}^{-2} \mathrm{~V}$ dose (Fig. 1), together with the presence of an abrupt photoresponse edge, is consistent with the presence of delocalized states within the Si gap. Therefore, we suggest that the observed photoconductivity may be related to carrier transitions involving the $\mathrm{CB}$ or the VB and the allowed states derived from the delocalization of the $\mathrm{V}$ deep levels.

Focusing our attention on the abrupt edge of the photoconductivity for the infrared light observed in the $10^{16} \mathrm{~cm}^{-2}$ $\mathrm{V}$ implanted sample, we have studied its behavior as a function of the temperature. Fig. 2 shows $\frac{\Delta G_{\square}}{I_{0}}$, as a function of the energy of the incident photons. The $\frac{\Delta G_{\square}}{I_{0}}$ is presented in a linear scale for different temperatures $(110 \mathrm{~K}, 90 \mathrm{~K}, 60 \mathrm{~K}$, $50 \mathrm{~K}$, and $35 \mathrm{~K}$ ). We observe that the photoconductivity edge shifts to lower photon energies as the temperature decreases. This observation suggests that the relative energy distance from the $\mathrm{CB}$ or the $\mathrm{VB}$ to the allowed states within the $\mathrm{Si}$

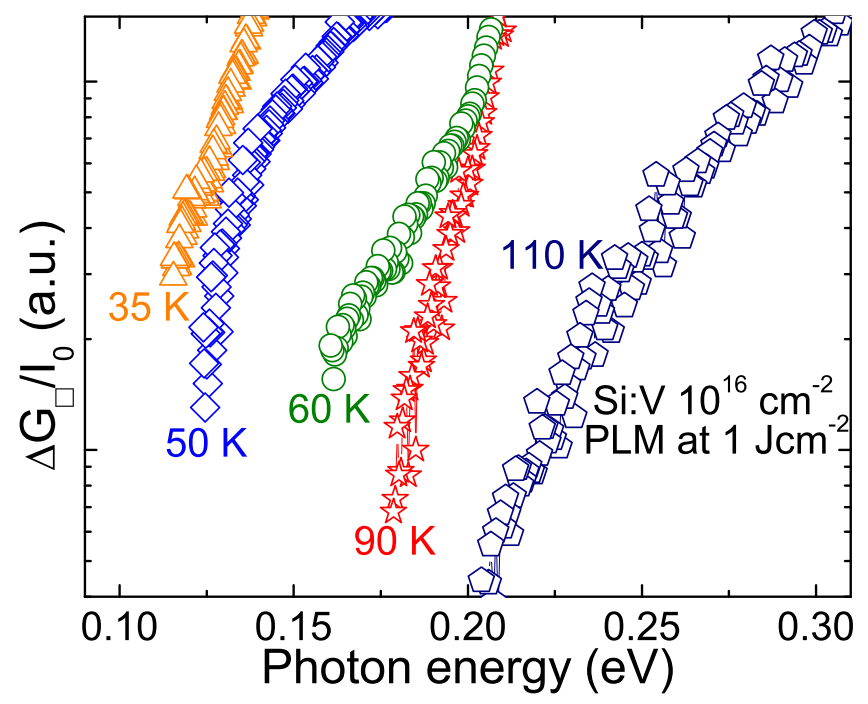

FIG. 2. Increase of the sheet conductance spectral response normalized to the impinging light power density as a function of the incident photon energy for the $\mathrm{V}$ implanted Si sample at $10^{16} \mathrm{~cm}^{-2}$ dose and PLM processed at $1 \mathrm{~J} \mathrm{~cm}^{-2}$. Measurements were carried at 35, 50, 60, 90, and $110 \mathrm{~K}$. gap is changing as a function of the temperature. This behavior is similar to the dependence of some semiconductors bandgap with the temperature. ${ }^{24}$

For a deeper understanding of the photoconductivity results, we have studied the structural characterization of the implanted and PLM samples. Fig. 3(a) shows the TEM images of the $\mathrm{V}$ implanted Si sample with the $10^{16} \mathrm{~cm}^{-2}$ dose and PLM at $1 \mathrm{~J} \mathrm{~cm}^{-2}$. In comparison, Fig. 3(b) shows the Si implanted Si sample at $10^{16} \mathrm{~cm}^{-2}$ dose and PLM at $1 \mathrm{~J} \mathrm{~cm}^{-2}$. Also high resolution TEM images and ED patterns are presented for both samples.

The V implanted sample at $10^{16} \mathrm{~cm}^{-2}$ dose (Fig. 3(a)) shows a slightly defective layer of $80 \mathrm{~nm}$, which is in the order of the depth that surpasses the Mott limit showed in Fig. S1 in the supplemental material $(100 \mathrm{~nm}) .{ }^{18}$ Focusing on the high resolution TEM image of this sample, we observe that a single crystalline layer is obtained in spite of the high $\mathrm{V}$ concentration. In addition, no differences between the ED pattern obtained for the processed layer and the ED pattern of a silicon reference substrate are observed. These results point to a very good crystal lattice reconstruction. This is a remarkable result since the solid solubility limit of $\mathrm{V}$ in $\mathrm{Si}\left(10^{15} \mathrm{~cm}^{-3}\right)^{25}$ has been surpassed in more than 6 orders of magnitude maintaining a high crystal quality. However, the Si implanted Si sample (Fig. 3(b)) shows a more defective layer of $150 \mathrm{~nm}$. A polycrystalline structure is observed in this defective layer. The high resolution TEM image of this sample shows details of a twin boundary. The ED pattern of this sample confirms the Si crystalline structure. The origin of this more defective layer is related to the different implantation energies $(170 \mathrm{keV}$ in the Si implanted sample and $35 \mathrm{keV}$ in the $\mathrm{V}$ implanted sample). We have chosen different implantation energies because our objective was to obtain a more defective layer in the case of the $\mathrm{Si}$ implanted Si sample. TEM images of the V implanted sample at $10^{13} \mathrm{~cm}^{-2}$ are shown in Fig. S2 in the supplemental material. ${ }^{18}$ For this sample, we have obtained a perfect

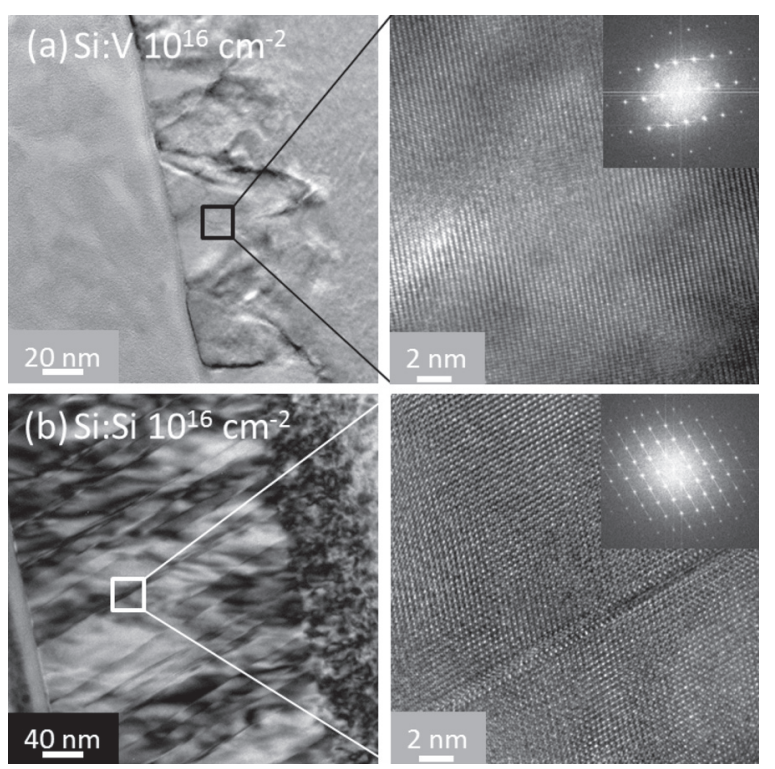

FIG. 3. Cross sectional TEM-HRTEM images and ED patterns of (a) the V implanted Si sample at $10^{16} \mathrm{~cm}^{-2}$ dose and (b) the Si implanted Si sample at $10^{16} \mathrm{~cm}^{-2}$ dose, and subsequently processed by PLM at $1 \mathrm{~J} \mathrm{~cm}^{-2}$. 
crystalline structure without differences with a Si reference substrate.

The different degrees of recrystallization that these samples present are directly related with the photoconductivity measurements of Fig. 1. In the case of the Si implanted Si, the structural defects observed in Fig. 3(b) could contribute to both the increase of the photoresponse, as it was previously observed in Si irradiated with electrons or neutrons in order to produce structural defects, ${ }^{26}$ and to the increase of the electrical noise due to the crystal disorder. ${ }^{23}$ However, the high photoresponse observed in the $\mathrm{V}$ implanted sample at $10^{16} \mathrm{~cm}^{-2}$ dose cannot be explained in terms of structural defects, since the most defective sample ( $\mathrm{Si}$ implanted $\mathrm{Si}$ sample) shows a much lower $\frac{\Delta G_{\square}}{I_{0}}$.

Regarding the dark sheet conductance at $90 \mathrm{~K}$ of the processed samples, they present values in the same order of magnitude: $0.93 \mathrm{mS}$ for the $\mathrm{V}$ implanted layer at $10^{16} \mathrm{~cm}^{-2}$ dose, $1.85 \mathrm{mS}$ for the $\mathrm{V}$ implanted layer at $10^{13} \mathrm{~cm}^{-2}, 1.42$ $\mathrm{mS}$ for the Si implanted layer at $10^{16} \mathrm{~cm}^{-2}$, and $1.68 \mathrm{mS}$ for the Si reference substrate. Measurements of the electrical transport as a function of temperature have shown important differences as a function of the $\mathrm{V}$ concentration as it can be seen in Fig. S3 in supplemental material. ${ }^{18}$ Samples with V concentration below the Mott limit show the same values as the Si reference substrate. However, samples with V concentration over the theoretical metal-insulator transition present an electrical decoupling effect of the bilayer structure formed by the implanted and PLM layer and the Si substrate. At low temperatures, these samples show a metallic transport behavior without a carrier concentration freeze-out effect that would be coherent with the proposed Mott transitions.

This electrical decoupling effect and metallic behavior is similar to the one observed in $\mathrm{Ti}$ supersaturated $\mathrm{Si}^{27,28}$ These electrical transport properties have been satisfactorily explained and reproduced by means of an analytical model which takes into account the parallel bilayer structure formed by the IB material/Si substrate. ${ }^{6}$ A detailed discussion of the electrical transport properties as well as the sheet conductance/temperature curve can be seen in supplemental material. ${ }^{18}$

Therefore, the higher photoresponse observed for the $\mathrm{V}$ implanted sample at $10^{16} \mathrm{~cm}^{-2}$ in Fig. 1 can be directly related to a V concentration over the Mott limit, which implies an important reduction of the electrical noise and the electrical decoupling effect observed in the transport properties.

In conclusion, $\mathrm{V}$ supersaturated $\mathrm{Si}$ samples have been obtained by means of ion implantation and PLM processes. Samples were implanted with two different V concentrations, below and above the theoretical metal-insulator transition limit. As the $\mathrm{V}$ concentration increases, an important increase of the photoresponse is observed for the far infrared region of the spectrum as well as an important reduction of the electrical noise. The infrared photoresponse of the $\mathrm{V}$ supersaturated $\mathrm{Si}$ samples exhibits a photoconductivity edge that shifts to lower photon energies as the temperature decreases. This photoresponse can neither be related with defects associated with the ion implantation nor with the PLM process, since a more defective Si implanted Si sample present a lower infrared photoresponse and a higher level of electrical noise. We have related the high intensity and far extended infrared photoresponse of the $\mathrm{V}$ sample implanted at $10^{16} \mathrm{~cm}^{-2}$ dose with the predictions of the delocalization of the deep levels associated with the V once the Mott limit concentration has been surpassed. This delocalization leads to a reduction of the NRR processes, which in turn is related to the reduction of the electrical noise and the formation of a band of allowed states in the Si bandgap. Therefore, an increase of both the charge carrier lifetime and the absorption coefficient as a consequence of sub-bandgap optical transitions involving this extra band takes place. These results could lead to the development of a future generation of Si based materials with enhanced photoresponse in the far infrared region of the spectrum.

Authors would like to acknowledge the CAI de Técnicas Físicas of the Universidad Complutense de Madrid for the ion implantations and metallic evaporations, the Nanotechnology and Surface Analysis Services of the Universidad de Vigo C.A.C.T.I. for ToF-SIMS measurements and the Instituto de Nanociencia de Aragón for the TEM images. This work was partially supported by the Project NUMANCIA II (Grant No. S-2009/ENE/1477) funded by the Comunidad de Madrid. Research by E. García-Hemme was also supported by a PICATA predoctoral fellowship of the Moncloa Campus of International Excellence (UCM-UPM). J. Olea and D. Pastor thank Professor A. Martí and Professor A. Luque for useful discussions and guidance and acknowledge financial support from the MICINN within the program Juan de la Cierva (JCI2011-10402 and JCI-2011-11471), under which this research was undertaken.

${ }^{1}$ P. R. Norton, Opt. Eng. 30, 1649-1663 (1991).

${ }^{2}$ A. Rogalski, Prog. Quantum Electron. 27, 59-210 (2003).

${ }^{3}$ C. H. Crouch, J. E. Carey, J. M. Warrender, M. J. Aziz, E. Mazur, and F. Y. Genin, Appl. Phys. Lett. 84, 1850-1852 (2004).

${ }^{4}$ A. J. Said, D. Recht, J. T. Sullivan, J. M. Warrender, T. Buonassisi, P. D. Persans, and M. J. Aziz, Appl. Phys. Lett. 99, 073503 (2011).

${ }^{5}$ J. Olea, M. Toledano-Luque, D. Pastor, G. González-Díaz, and I. Mártil, J. Appl. Phys. 104, 016105 (2008).

${ }^{6}$ J. Olea, G. González-Díaz, D. Pastor, I. Mártil, A. Martí, E. Antolín, and A. Luque, J. Appl. Phys. 109, 063718 (2011).

7J. Olea, Á. del Prado, D. Pastor, I. Mártil, and G. González-Díaz, J. Appl. Phys. 109, 113541 (2011).

${ }^{8}$ N. F. Mott, Adv. Phys. 21, 785-823 (1972).

${ }^{9}$ C. H. Henry and D. V. Lang, Phys. Rev. B 15, 989-1016 (1977).

${ }^{10}$ A. Luque, A. Martí, E. Antolín, and C. Tablero, Phys. B: Condens. Matter 382, 320-327 (2006).

${ }^{11}$ D. Pastor, J. Olea, Á. del Prado, E. García-Hemme, R. García-Hernansanz, and G. González-Díaz, Sol. Energy Mater. Sol. Cells 104, 159-164 (2012).

${ }^{12}$ M. T. Winkler, D. Recht, M.-J. Sher, A. J. Said, E. Mazur, and M. J. Aziz, Phys. Rev. Lett. 106, 178701 (2011).

${ }^{13}$ E. Antolín, A. Martí, J. Olea, D. Pastor, G. González-Díaz, I. Mártil, and A. Luque, Appl. Phys. Lett. 94, 042115 (2009).

${ }^{14}$ E. García-Hemme, R. García-Hernansanz, J. Olea, D. Pastor, Á. del Prado, I. Mártil, and G. González-Díaz, Appl. Phys. Lett. 101, 192101 (2012).

${ }^{15}$ T. Sadoh, H. Nakashima, and T. Tsurushima, J. Appl. Phys. 72, 520-524 (1992).

${ }^{16}$ C. Goletti, G. Bussetti, P. Chiaradia, and G. Chiarotti, J. Phys. Condens. Matter 16, S4289-S4300 (2004).

${ }^{17}$ W. Muller and W. Monch, Phys. Rev. Lett. 27, 250 (1971).

${ }^{18}$ See supplementary material at http://dx.doi.org/10.1063/1.4813823 for the figure of the $\mathrm{V}$ concentration depht profile figure, TEM images of the $\mathrm{V}$ implanted Si sample at $10^{13} \mathrm{~cm}^{-2}$ dose, figure of the dark sheet conductance as a function of the temperature as well as for some discussion of electrical transport properties. 
${ }^{19}$ H. Indusekhar and V. Kumar, Phys. Status Solidi A 95, 269-278 (1986). ${ }^{20}$ A. C. Wang, L. S. Lu, and C. T. Sah, Phys. Rev. B 30, 5896-5903 (1984).

${ }^{21}$ L. K. J. Vandamme, IEEE Trans. Electron Devices 41, 2176-2187 (1994).

${ }^{22}$ W. A. Beck, Appl. Phys. Lett. 63, 3589-3591 (1993).

${ }^{23}$ F. N. Hooge, IEEE Trans. Electron Devices 41, 1926-1935 (1994).

${ }^{24}$ R. Dalven, Phys. Rev. B 8, 6033-6034 (1973).
${ }^{25}$ H. H. Woodbury and G. W. Ludwig, Phys. Rev. 117, 102-108 (1960).

${ }^{26}$ R. C. Young and J. C. Corelli, Phys. Rev. B 5, 1455 (1972).

${ }^{27}$ J. Olea, G. González-Díaz, D. Pastor, and I. Mártil, J. Phys. D 42, 085110 (2009).

${ }^{28}$ D. Pastor, J. Olea, Á. del Prado, E. García-Hemme, R. García-Hernansanz, I. Mártil, and G. González-Díaz, J. Phys. D 46, 135108 (2013). 